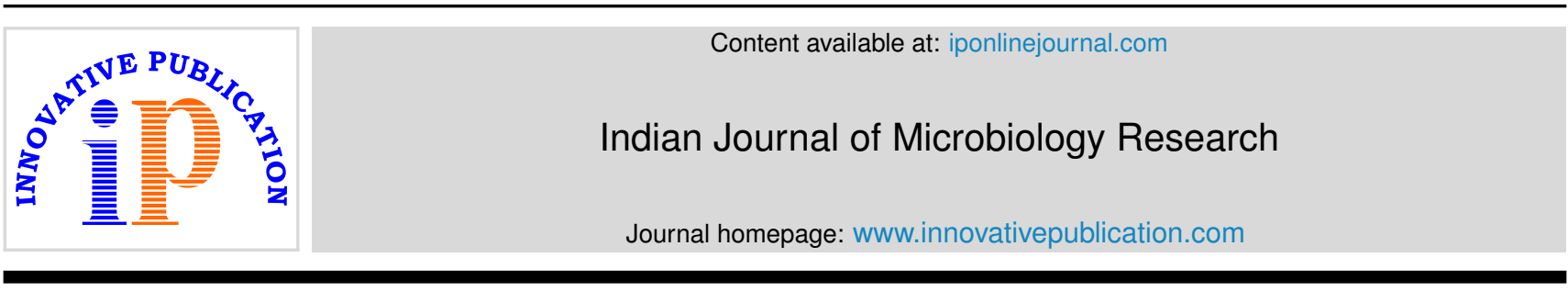

Original Research Article

\title{
Pandemonium of oral flora in tobacco chewers and patients with associated oral cancer in a tertiary care hospital, Valsad, South Gujarat
}

\author{
Adarsh Sunil Patil ${ }^{1} *$, Alka B Nerurkar ${ }^{1}$, Payal Soni ${ }^{1}$, Parimal Patel ${ }^{1}$ \\ ${ }^{1}$ Gmers Medical College, Valsad, Gujarat, India
}

\section{A R T I C L E I N F O}

\section{Article history:}

Received 26-03-2019

Accepted 24-07-2019

Available online 09-09-2019

Keywords:

Oral flora

Tobacco chewers.

\begin{abstract}
A B S T R A C T
Introduction: Tobacco chewing is a serious oral health problem in India responsible for halitosis, periodontitis, sub mucus fibrosis, leukoplakia and oral cancer. There is a need to understand the oral microbiological changes in tobacco chewers.

Aim and Objective: To find out the changes in oral microbiota in tobacco chewing patients and patients with associated oral cancer.

Materials and Methods: A case control study on 102 patients was conducted over a period of 2 months. Case group included subjects with tobacco chewing habits and presence of leukoplakia and oral cancer while control group included subjects with no tobacco chewing habits. Oral swabs collected were microscopically examined and grown on routine culture media- Blood Agar, Nutrient Agar and McConkey's Agar. After obtaining the complete data of different bacteria isolated, a statistical analysis was made comparing the isolates from case and control group.

Results: Streptococci spp. and Coagulase Negative Staphylococcispp (CONS) forms a part of normal oral flora, which was found in $79 \%$ of the control isolates but only in $49 \%$ of the case isolates. This result of normal flora being reduced in case group was statistically significant $(\mathrm{p}<0.05)$. Similarly, in the control group, other species- Enterococci, Pseudomonas and Klebsiella were found in only $21 \%$ of control isolates while $51 \%$ of cases showed such result but Acinetobacter spp, Proteus spp and Citrobacter freundii were also isolated.

Conclusion: Tobacco chewing is associated with a disruption of normal oral flora and a consequent increase in other organisms making the oral cavity more prone to ailments.
\end{abstract}

(c) 2019 Published by Innovative Publication.

\section{Introduction}

Walter Scott had rightly stated- “ Tobaccos but an Indian weed; grows green at morn, cut down at eve. It shows our decay; we are but clay." Everyday almost 2500 people quit tobacco in India - By Dying! Global Adult Tobacco Survey (GATS) conducted by the World Health Organization reveals that $25.9 \%$ of Indian adults are the users of smokeless tobacco ${ }^{1}$ - the most common form of consumption being tobacco, paraffin wax, arecanut and slaked lime. It is a practice among tobacco chewers in India to place the quid in the inner side of the lower lip.

\footnotetext{
* Corresponding author.

E-mail address: adarsh4smile@gmail.com (A. S. Patil).
}

The consumers of tobacco chew it under many impressionssome say it is euphoric while others say it makes their teeth stronger. Many patients even believe that tobacco eliminates their pyorrhea. But the other dark side of this silent killer is probably still unknown to them.

The deleterious effects of tobacco are quite remarkablethe most menacing being halitosis, periodontitis, submucous fibrosis, leukoplakia and oral cancer. Consumption of tobacco during pregnancy is also implicated in still births. ${ }^{2}$ Nitrosamines, polycyclic aromatic hydrocarbons, nitrosodietheinalamine and nitrosoproline are the chemical constituents of tobacco that are toxic to the mucosa of the oral cavity and thus a cause of oral lesions. ${ }^{3}$ Betel chewing produces reactive oxygen species (ROS) and can be 
directly involved in the tumor initiation process by inducing mutations in p53 and RB tumor suppressor genes. ${ }^{4,5}$ In fact, chewing of betel quid in itself is declared to be group 1 carcinogen. ${ }^{6}$

India has one of the highest rates of oral cancer in the world. In south Gujarat too, tobacco users are well prevalent. A study conducted on such 504 patients in Surat concluded statistical increase in oral cancer in lower age group and anterior oral cavity showed involvement in $61.3 \%$ of the cases. ${ }^{7}$ In our tertiary healthcare setup, majority of the patients are tobacco chewers.

Amidst such social and medical predicaments, there is also a serious need for understanding the widespread microbiological changes occurring in the oral cavity. Carcinogenicity has only been attributed to the toxic compounds present in the tobacco quid, but very little has been known about its microbiological role. The role of bacteria in the etiopathogenesis of oral cancer has not been decoded but the continuous presence of them at the tumor sites raises intriguing questions and the door to understand the mysterious power of these bugs is yet to be completely opened. Ongoing studies indicate that tobacco chewing is associated with alteration of normal oral residential flora and this might be a contributing factor for the development of oral lesions. The gap in this enigma is yet to be discerned, whether the toxic metabolites itself induce the damage or the change in the oral flora does or may be a synergistic action is responsible.

The aim of this study is therefore to find out the changes in oral microbiota in tobacco chewers and patients with oral cancer.

\section{Materials and Methods}

Before beginning the study, Ethical clearance was duly obtained from the Human Institutional Ethics committee (HIEC).

A prospective case-control study was carried out over a period of 2 months as a part of STS (Short Term Studentship) programme under ICMR in the Microbiology Laboratory at a Tertiary Care Hospital, Valsad. The specimens were obtained from the outpatients and inpatients visiting the ENT, Medicine and Surgery departments of the hospital. Case specimens were obtained from the subjects with chronic tobacco chewing habit and also subjects with associated oral cancer while control specimens were obtained from subjects who never consumed tobacco.

\subsection{Study Population}

102 patients.

\subsection{Inclusion criteria}

Subjects falling under the following criteria
1. Males and females of age group: 15-70 years.

2. Tobacco chewing habit of minimum one-year duration and consuming at least 2-3 quids per day.

3. Presence of leukoplakia, periodontitis and oral cancer.

4. With no tobacco $\mathrm{c}$ hewing habits as control group.

After taking an informed oral consent from the subjects, oral swab specimens were obtained by scrubbing two sterile swabs at the following sites: lateral sides of the tongue, buccal epithelium and mandibular anterior vestibule. The specimens been put in a sterile container were properly labelled and transported to the Microbiology laboratory, where it underwent microscopic examination in accordance with the Standard Gram Staining techniques. Routine Culture media appropriate for the growth of bacteria were chosen like Blood Agar, Nutrient Agar and McConkey's Agar. The medias were inoculated and incubated under specific aerobic and anaerobic conditions at 37 degrees for 18-24 hours. The bacteria were then identified as per standard microbiological procedures. ${ }^{8}$ Growth was observed and Gram staining was done on individual colonies to confirm gram positive and gram negative bacteria. The number of bacterial colonies on the culture plates was noted and their morphology was studied.

Specific biochemical tests to further differentiate and identify the bacteria were performed. For gram positive cocci - Catalase and coagulase tests.

For gram negative cocci-Triple sugar iron test, Sugar fermentation, Citrate, Indole, Phenylpyruvic acid(PPA) and urease tests.

The biochemical tests were read, optochin sensitivity to differentiate Streptococcus spp was done and the different isolated organisms were noted. After obtaining the complete data of different bacteria isolated from all the specimens, a statistical analysis was made on Excel platform categorizing them according to control and case subjects. Tests for significance was done by Chi-square method on Epi Info7 platform.

\section{Results}

Out of the total 102 specimens collected, 38 belonged to the subjects who never consumed tobacco i.e. Control group. 64 belonged to the subjects who had chronic tobacco chewing habits of minimum 1 year duration i.e. Case group out of which 11 specimens belonged to the subjects with associated oral malignancy.

Of the 38 control specimens, 47 isolates were obtained while in the case group of 64 specimens, 88 isolates were obtained.

Males predominated the females with habits of tobacco chewing. Majority of the case subjects were chewing tobacco for more than 10 years, some even for 50 years. $71 \%$ of the case subjects gave the history of chewing tobacco for more than 10 years. 
Table 1: Number and percentage of organisms isolated from the control group.

\begin{tabular}{ll}
\hline \multicolumn{2}{l}{ Organisms isolated from the control group. } \\
Organism & Number of isolates and percenatge \\
Streptococcus spp & $(31)-66 \%$ \\
CONS & $(06)-13 \%$ \\
Pseudomonas & $(03)-06 \%$ \\
Acinetobacter & $(01)-02 \%)$ \\
Enterococcus & $(04)-09 \%$ \\
E. Coli & $(01)-02 \%$ \\
Klebsiella & $(01)-02 \%$ \\
\hline
\end{tabular}

(CONS = Coagulase negative Staphylococci

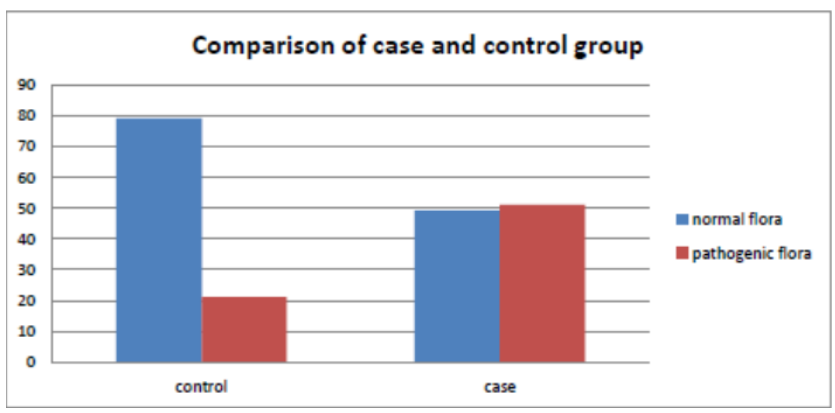

Fig. 1: Chart depicting the comparison between the caseand the control group with respect to the percentage of the normal flora andthe other organisms isolated. Note the relative decrease of normal flora andincrease in other organisms in the case group as compared to the control group

Table 2: Number and percenatge of organisms isolated from the case group.

\begin{tabular}{lc}
\hline $\begin{array}{l}\text { Organisms isolated from the Case group } \\
\text { Organism }\end{array}$ & $(30)-34 \%$ \\
Streptococcus spp & $(13)-15 \%$ \\
CONS & $(12)-14 \%$ \\
Klebsiella & $(12)-14 \%$ \\
Pseudomonas & $(10)-11 \%$ \\
Enterococcus & $(04)-04 \%$ \\
Acinetobacter & $(02)-02 \%$ \\
Edwardsiella & $(01)-1.2 \%$ \\
Peptostreptococcus & $(02)-2.4 \%$ \\
E. coli & $(01)-1.2 \%$ \\
Proteus & $(01)-1.2 \%$ \\
Citrobacter freundii & \\
\hline
\end{tabular}

It is interesting to note in our result that, out of the 11 specimens of case subjects who are chronic tobacco chewers with associated oral malignancy, 14 isolates were obtained out of which only 2 isolates belonged to Streptococcus spp while the remaining 12 belonged to others, here too with a wide range of organisms- Edwardsiella,Acinetobacter, Pseudomonas, Enterococci and Klebsiella.

\section{Discusssion}

The above results indicate that there is a change in the microbiology of the oral cavity in the tobacco chewers and also in subjects with associated oral malignancy.

Streptococcus and Coagulase Negative Staphylococci were the most frequently isolated organisms. These constitute the " normal flora' of the oral cavity. The organisms isolated other than these were the environmental pathogens.

According to the results, $79 \%$ of the isolates from control specimens showed the presence of normal flora, while only $49 \%$ of isolates from the cases showed a similar result a difference of almost $30 \%$. The result of normal flora being reduced in cases compared to controls was significant $(\mathrm{p}<0.05)$ Thus, there is a progressive decrease in the normal residential oral flora with tobacco chewing habits. In a similar study by Saini S. et al, out of the total 25 specimens collected in each case and control group, Streptococcus spp was found in only $20 \%$ of the cases while the controls showed $75 \%$. $^{3}$ Thus, tobacco not only has deleterious effect on the mucosa of the oral cavity, but chewing it on a chronic basis might make the microenvironment of the oral cavity unsuitable for the normal residential flora to reside there and hence decreasing their population.

In the control group, microbes other than the normal flora-Enterococci, Pseudomonas and Klebsiella was found in only $21 \%$ of the controls. These results in the control group might be due to unhygienic conditions while $51 \%$ of the cases showed similar result - a difference here too of almost $30 \%$ - but the bacteria isolated in the case group being Klebsiella, Acinetobacter, Pseudomonas, Proteus and Citrobacter freundii. The result of other organisms being increased in cases compared to controls was significant $(p<0.05)$. Therefore, the decrease in the normal flora of the oral cavity may derange the beneficial effects they have and instead clear the way for other organisms to intrude and initiate the deleterious effects including infection and tumorigenesis. Sudha Sellappa et al in their study also found increased bacterial growth in tobacco chewers and smokers compared to controls. ${ }^{9}$

Considering the fact about the role of bacteria in tumorigenesis, Naggy et al in their study found that Serratia, Klebsiella pneumoniae and Citrobacter freundii were more frequently found at the tumor sites than the non-tumor sites. In our study too, Citrobacter freundii and Klebsiella were isolated in the case group, but a conclusive result cannot be established.

In our study, males had preponderance over females with tobacco chewing habits. In the case group, $97 \%$ were males while only $3 \%$ were females who had tobacco chewing habits. Therefore, males are more likely to suffer from periopathogenic infections. Tobacco being a significant risk factor for oral cancer, therefore has a predilection for males. This is also in well accordance to the Global epidemiology 
report of oral cancer by Warnakulasuriya. ${ }^{10}$

\section{Conclusion}

The habit of tobacco chewing is highly prevalent in our country induces widespread changes in the oral cavity. It is concluded by our study that tobacco chewing causes reduction in the normal flora and increase in the other organisms. This is just the tip of the iceberg that we see, many questions now arise. Are there any specific bacteria responsible for the oral ailments by tobacco? If so, can oral malignancies be also developed as a result of tumorigenesis by such bacteria? Can these microbiological changes in the oral cavity be of any value in the early diagnosis of oral ailments? All this creates a need for much more deep exploration of plethora of these questions.

\section{Source of Funding}

None.

\section{Conflict of Interest}

None.

\section{References}

1. Global adult tobacco survey (GATS) fact sheet India ;.

2. Suliankatchi RA, Sinha DN. The Human Cost of Tobacco Chewing Among Pregnant Women in India: A Systematic Review and Metaanalysis. J Obstet Gynaecol India. 2016;66(1):161-166. Suppl. Available from: 10.1007/s13224-015-0821-7.
3. Saini S, Saini SR, Katiyar R, Bhalerao DS. Munde A The use of tobacco and betel leaf and its effect on the normal micro flora of oral cavity. Pravara Med Rev. 2009;1(4):17-19.

4. Anand R, Dhingra C, Prasad S, Menon I. Betel nut chewing and its deleterious effects on oral cavity. J Can Res Ther. 2014;10:499-505.

5. Mubeen K, Chandrashekhar H, Kavitha M, NS. Effect of Tobacco on oral-Health an Overview. J Evol Med Dent Sci. 2013;2:3523-3534.

6. YJC, JTCC, CTL, HMW, TCY, editors ;.

7. An- Relationship of Oral cancer with age, sex, site, distribution and habits. Indian J Pathol Microbiol. 2004;47(2):195-202.

8. Jawetz M. -. Medical Microbiology;p. 742-745. 26th Edition.

9. Sudha Selappa et al : Association of bacterial growth in the oral cavity between tobacco smokers and chewers. International Journal of Toxicological and pharmacological Research. 2015;7(4):228-231.

10. Warnakulasuriya S. et al - Global Epidemiology of oral and oropharyngeal cancer. Oral Oncol. 2008;45:309-325. Available from: 10.1016/j.oraloncology.2008.06.002.Epub.

\section{Author biography}

Adarsh Sunil Patil Under Garaduate

Alka B Nerurkar Professor and HOD

Payal Soni Assistant Professor

Parimal Patel Assistant Professor

Cite this article: Patil AS, Nerurkar AB, Soni P, Patel P. Pandemonium of oral flora in tobacco chewers and patients with associated oral cancer in a tertiary care hospital, Valsad, South Gujarat. Indian J Microbiol Res 2019;6(3):186-189. 